\title{
COMPUTATIONAL STUDY OF DIFFUSER AUGMENTED WIND TURBINE USING ACTUATOR DISC FORCE METHOD
}

\author{
V.V. DIGHE, F. AVALLONE \& G.J.W. VAN BUSSEL \\ TU Delft, Delft, Netherlands, 2629HS.
}

\begin{abstract}
In this paper, a computational approach, based on the solution of Reynolds-averaged-Navier-Stokes (RANS) equations, to describe the flow within and around a diffuser augmented wind turbine (DAWT) is reported. In order to reduce the computational cost, the turbine is modeled as an actuator disc (AD) that imposes a resistance to the passage of the flow. The effect of the $\mathrm{AD}$ is modeled applying two body forces, upstream and downstream of the $\mathrm{AD}$, such that they impose a desired pressure jump. Comparison with experiments carried out in similar conditions shows a good agreement suggesting that the adopted methodology is able to carefully reproduce real flow features.

Keywords: actuator disc simulation, CFD, diffuser augmented wind turbine.
\end{abstract}

\section{INTRODUCTION}

The impetus towards a sustainable and environmental friendly world has led the wind energy industry to grow, bringing the global total production to $369.6 \mathrm{GW}$ (2014); a new record of more than $51 \mathrm{GW}$ installed in a single year [1]. Wind energy is abundant, and therefore, a wind energy system that intensifies the power production without the size penalty has long been sought. The use of diffusers or ducts to enhance the performance of wind and tidal turbines have been proposed and discussed for many years now [2-6]. The basic idea is that the presence of a diffuser increases the mass flow across the blades, thus generating more thrust. It has been proved experimentally by several manufacturers that this practice increases the wind turbine performances when compared with the bare configuration [7, 8]. Some independent studies, using primarily computational fluid dynamics (CFD), suggest a substantial rise in power production up to three times more than the bare turbine [9]. Nevertheless, details of these studies are not made available on public domain.

Since computations are widely used during the design process, it is always necessary to develop new faster approaches and prove that they are reliable. This paper presents a 2D CFD study of the 'donQi Urban Windmill 1.5' diffuser augmented wind turbine model (DAWT) [10] using the commercial solver Ansys FLUENT. The turbine is modeled as an actuator disc (AD), since it has been proved experimentally that this method is able to reproduce the near wake flow for a bare wind turbine [11]. The influence of $\mathrm{AD}$ in the equations can be implemented as an additional body force acting against the flow. The most common approach reported in the literature is to create an interface where a momentum sink term is applied [12, 13]. The associated pressure drop $(\Delta p)$ is calculated from a semi-empirical relation between the thrust coefficient curve and the velocity at the disc. While these simulations provide a good estimate for the far wake velocity profiles, the flow behavior in front of the AD and in the near wake is not captured accurately [12]. The discrepancies between computed velocity profiles and real measurements may be attributed to the way the $\Delta p$ is distributed over several cells. Numerically, the force is smeared over the neighboring cells affecting the flow in the local position of the $\mathrm{AD}[12]$. 
In order to correctly describe the flow in the near wake and in proximity of the AD, a discrete force allocation technique, called the AD force method is used [14]. Discrete body forces are, in the present analysis, used to model the velocity deficit across the AD inside the diffuser. This method redistributes the forces over the neighboring cells of the $\mathrm{AD}$, and then it derives the corresponding cell faces pressure jump. A detailed explanation of the methodology is presented in Section 3.2

\section{WIND TUNNEL EXPERIMENT}

Experiments were conducted in the closed-loop open-jet wind tunnel facility at the Delft University of Technology. The tunnel has the maximum speed of $35 \mathrm{~m} / \mathrm{s}$ and an octagonal outflow nozzle of $2.85 \mathrm{~m} \times 2.85 \mathrm{~m}$. The free-stream flow at the measurement location has a turbulent intensity of approximately $0.21 \%$ [15]. The flow temperature is kept constant at $20^{\circ} \mathrm{C}$, which is achieved by a $350 \mathrm{~kW}$ heat exchanger.

A metal porous disc with a diameter of $1.5 \mathrm{~m}$ (Fig. 1) was manufactured in order to mimic the energy extractor at the turbine blade location. The AD has a uniform thickness of 0.002 $\mathrm{m}$ with porosity, $\varnothing=42 \%$, resulting in an average thrust coefficient, $C_{T}=0.87$ [15]. The porosity is defined as the ratio of open area and the total area of the disc and it is a measurement of the permeable area of the AD.

The pressure jump across the bare $\mathrm{AD}$ area produces a thrust force, $F_{A D}$ The presence of the diffuser increases the mass flow passing through the AD thus resulting in a larger thrust force $F_{D A W T}$. Both these values were measured using a six component balance (Table 1). Furthermore, pitot-static measurements were used to measure the velocity distribution along the axial $(x)$ and the radial $(y)$ directions; distances indicated in Figure 2.

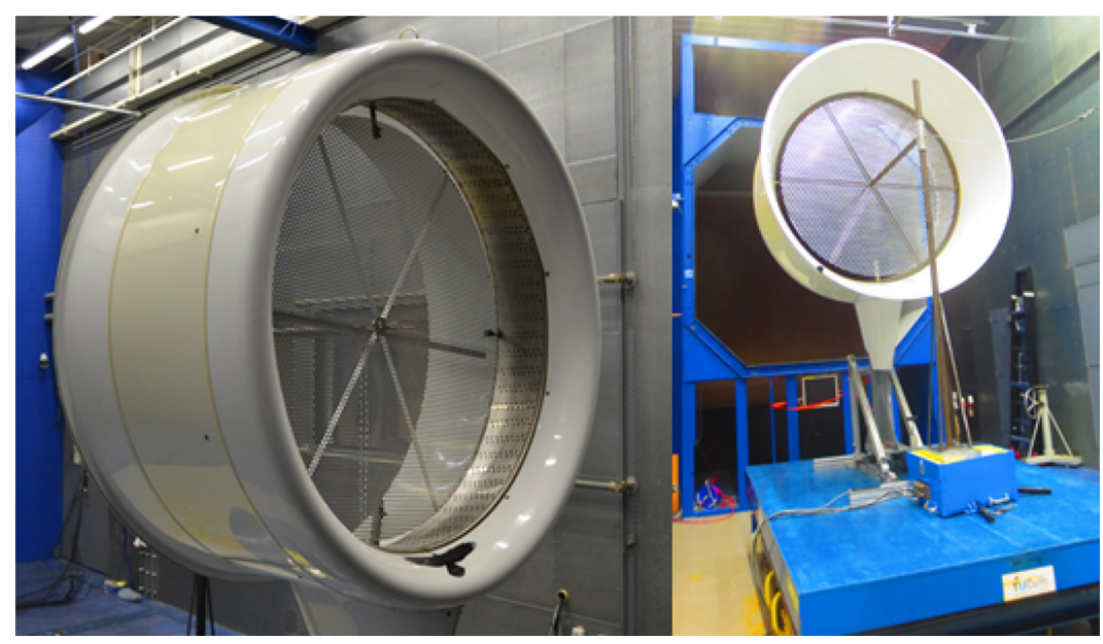

Figure 1: Pictures showing the experimental setup. (left) Zoom of the AD located within the diffuser; (right) view of the entire setup with the load balance used to measure the thrust of the AD. 
Table 1: Load cell measurements for different free stream velocities.

\begin{tabular}{lll}
\hline$U_{\infty}(m / s)$ & $F_{A D}(N)$ & $F_{A D W T}(N)$ \\
\hline 5 & 22.3 & 31 \\
7 & 45.2 & 84 \\
10 & 94.2 & 131 \\
\hline
\end{tabular}

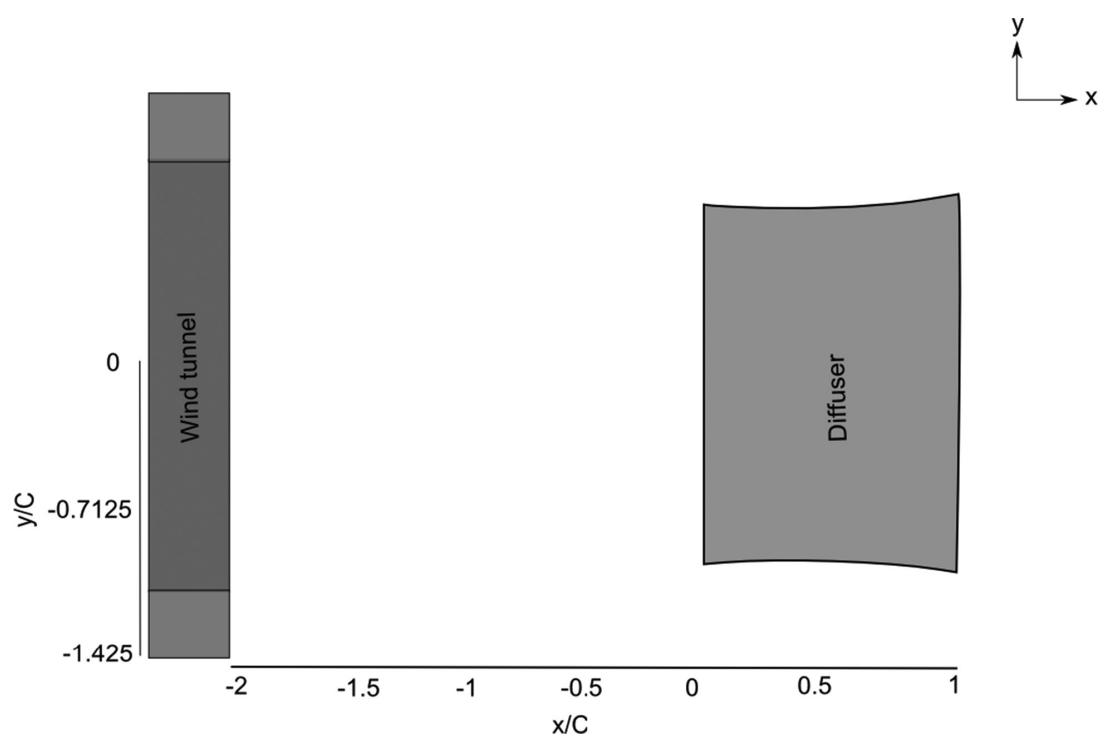

Figure 2: Schematic of experimental set-up showing measurements at diffuser locations.

\section{CFD METHODOLOGY}

The following section presents the description of the CFD methodology adopted in this paper. A detailed description of the AD modeling following the approach suggested by Réthoré et al. [14] is reported in this section.

\subsection{Computational domain}

A computational domain similar to the experimental configuration was built using ICEM CFD, Ansys Inc. A C-grid structured mesh was chosen to discretize the domain (Fig. 3), which proved advantageous in the case of a curved boundary (diffuser leading edge), and terminating the $\mathrm{C}$-shaped loop in the wake region. The final mesh consisted of 78,145 elements, which was achieved after a mesh independence study; this minimizes the computational power and the time required for the generation of a grid independent solution. Even if 2D, the investigated configuration represents well the experimental setup because of the axis-symmetric flow conditions. 


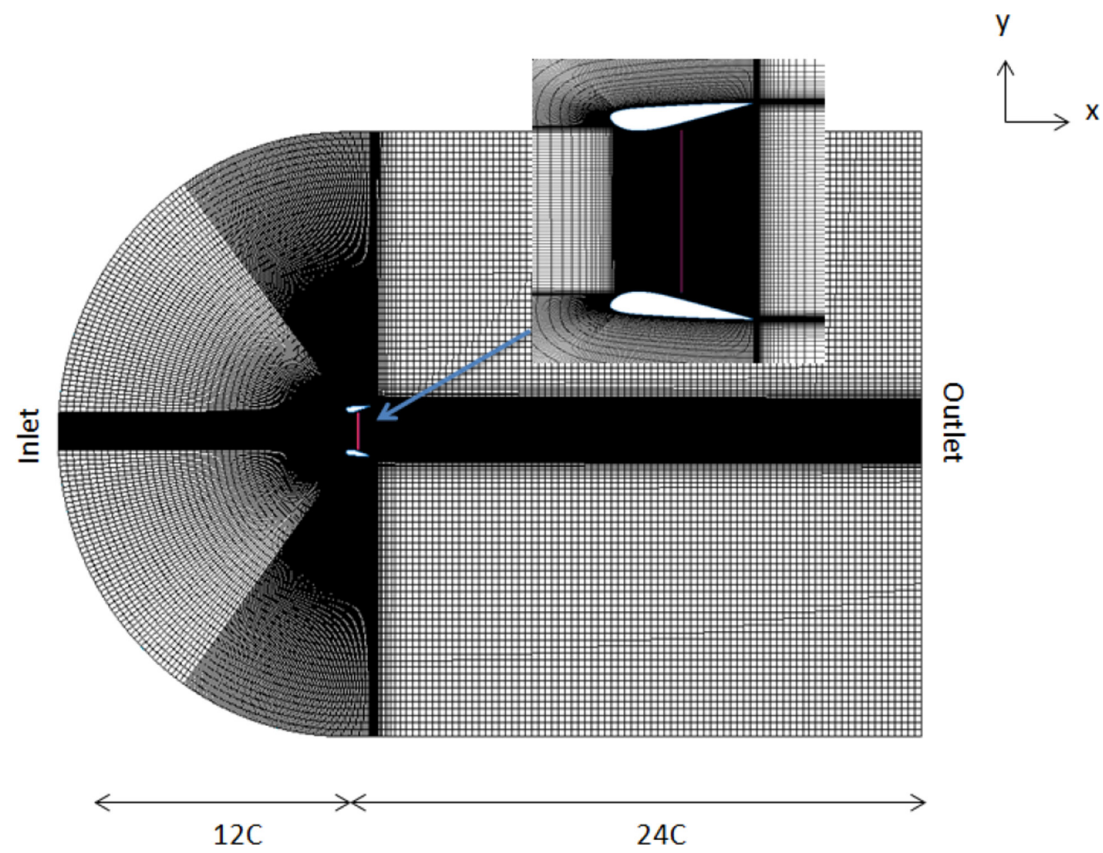

Figure 3: Computational mesh visualizing the $\mathrm{AD}$ within the diffuser.

\subsection{Actuator disc modeling}

The main aim of the present paper is to find a fast and reliable approach to solve the flow within and around a DAWT by solving the RANS equations, which can be used for performance analysis of the DAWT in future work. Previous studies have shown that overestimation of the velocity inside the diffuser with consequent overestimation of the power extracted from the DAWT [16]. Therefore, it is necessary to have a computational method, which reproduces a realistic velocity profile inside the diffuser in presence of a turbine (i.e. AD in this context). To reach this goal, the AD force method was used [14] since it has been proved to correctly model the bare wind turbines [17]; however, it is not clear how this method copes with the presence of a diffuser. In the following, discrete body forces are defined at the face of the cells in order to introduce a desired pressure jump $(\Delta p)$. The body force in the cell representing the AD is splitted into two pressure jumps: one on the upstream face $U$ and one on the downstream face $\mathrm{D}$, as shown in Figure 4. The approach consists of defining the normal force acting over the $\mathrm{AD}$ area on the upstream face $U$, which is kept constant:

$$
F_{A D}=\frac{1}{2} \rho C_{T} A U_{\infty}^{2}
$$

with $\rho$ as the air density, $C_{T}$ as the thrust coefficient, $A=\pi R^{2}$ as the rotor area with $\mathrm{R}$ as the $\mathrm{AD}$ radius and $U_{\infty}$ as the ambient velocity. The total pressure drop encountered for a DAWT under the influence of $\mathrm{AD}$ would take into account an additional force, $F_{\text {diffuser }}$, and the total force would be specified as a pressure term on face $D$ 

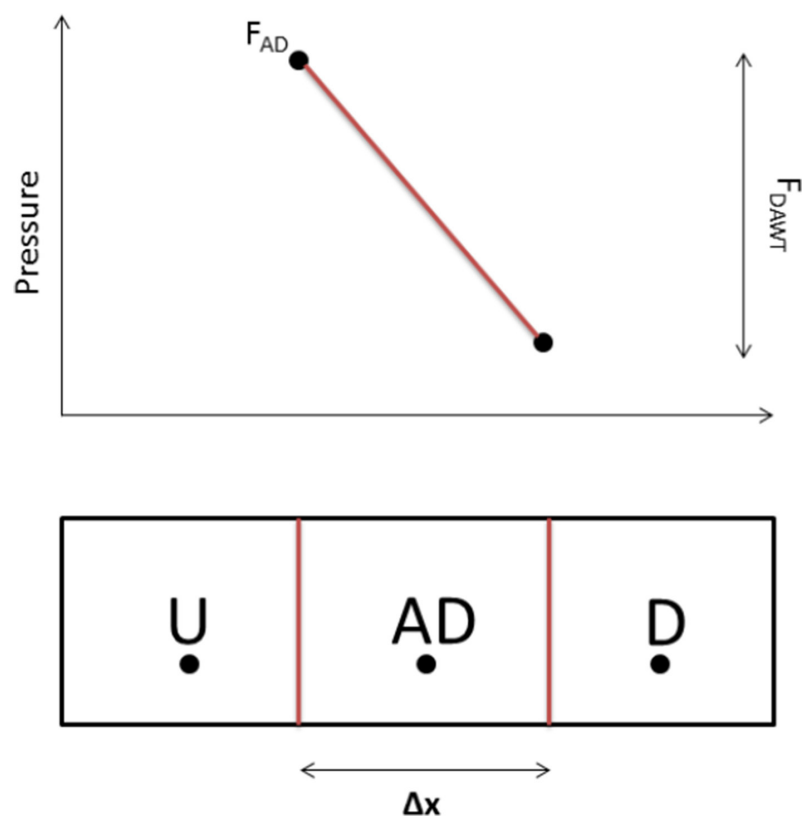

Figure 4: Schematic representation of pressure drop using the AD force method.

$$
\begin{gathered}
F_{D A W T}=F_{A D}+D a F_{\text {diffuser }}, \\
\Delta p=\frac{F_{D A W T}}{A} .
\end{gathered}
$$

\subsection{Turbulence modeling}

The maximum free stream velocity set during the experiments does not exceed $10 \mathrm{~ms}^{-1}$; leading to incompressible flow conditions for the CFD simulations. The inlet parameters were pre-determined to produce matching flow conditions, including the turbulent intensity. To appropriately select a turbulence model to compute the flow around a DAWT, a study was conducted for initial validation. Two turbulence models, $k-\varepsilon$ and $k-\omega$ are widely used for AD CFD simulations due to their relatively low computational power requirements and better stability in reaching convergence [18]. Both the models have their advantages, for instance, the $k-\omega$ is more accurate in formulating the near-wall region flows, whereas $k-\varepsilon$ has a free stream independence in the far-field region. In order to replicate the flow correctly around and within the DAWT, the overall SST $k-\omega$ model was adopted; it applies the $k-\omega$ solutions in the inner part of the boundary layer and then switches to $k-\varepsilon$ to resolve the far-field with the inlet turbulence properties.

\subsection{Blockage correction}

In this numerical study, the DAWT faces an undisturbed free stream flow. However, in order to compare with wind tunnel experiments, the effect of the blockage needs to be accounted 
for since it results in a free stream velocity higher than the one expected in presence of an undisturbed free stream flow. A common approach is to use a blockage correction factor based on the dominant flow effects as outlined below.

Mercker and Wiedemann [19] have identified the major blockage causes in presence of open-jet and have developed procedures to correct their effects. While a rigorous analysis of these effects is beyond the scope of this paper, an overview of the method used is presented. The correction methodology breaks the boundary-induced effects into series of sub components that are combined to correct the dynamic pressure measurements recorded by the pitot-static tube.

$$
\frac{q_{c}}{q_{u}}=\left(1+\varepsilon_{s}+\varepsilon_{n}+\varepsilon_{c}\right)^{2}
$$

where $\frac{q_{c}}{q_{u}}$ is the total blockage correction factor, and is made up of solid blockage, $\varepsilon_{s}$, which is negative, the nozzle blockage, $\varepsilon_{n}$, which is positive, and the collector blockage factor, $\varepsilon_{c}$, which is also positive. The factor was calculated to be 0.96 , which was used to correct the experimental dynamic pressure measurements.

\section{RESULTS AND DISCUSSION}

In the following, experimental data are used to validate the CFD approach, which has been done by comparing the velocity profiles inside the diffuser. Three free stream velocity equal to 5, 7 and $10 \mathrm{~ms}^{-1}$ were investigated for an $\mathrm{AD}$ with a constant loading, $C_{T}=0.87$.

In the present analysis, the location of the $\mathrm{AD}$ inside the diffuser is at $x / C=0.5$. The presence of the $\mathrm{AD}$ causes a drop of the static pressure; in order to accurately reproduce the flow condition, the correct pressure drop needs to be estimated. Figure 5 displays the computed pressure distribution along the diffuser center-line for different $U_{\infty}$. The pressure shows a strong jump at $\mathrm{x} / \mathrm{C}=0.5$ (the location of the $\mathrm{AD}$ ), which is based on the input parameters specified using the $\mathrm{AD}$ force method. It is evident that the static pressure far upstream and far

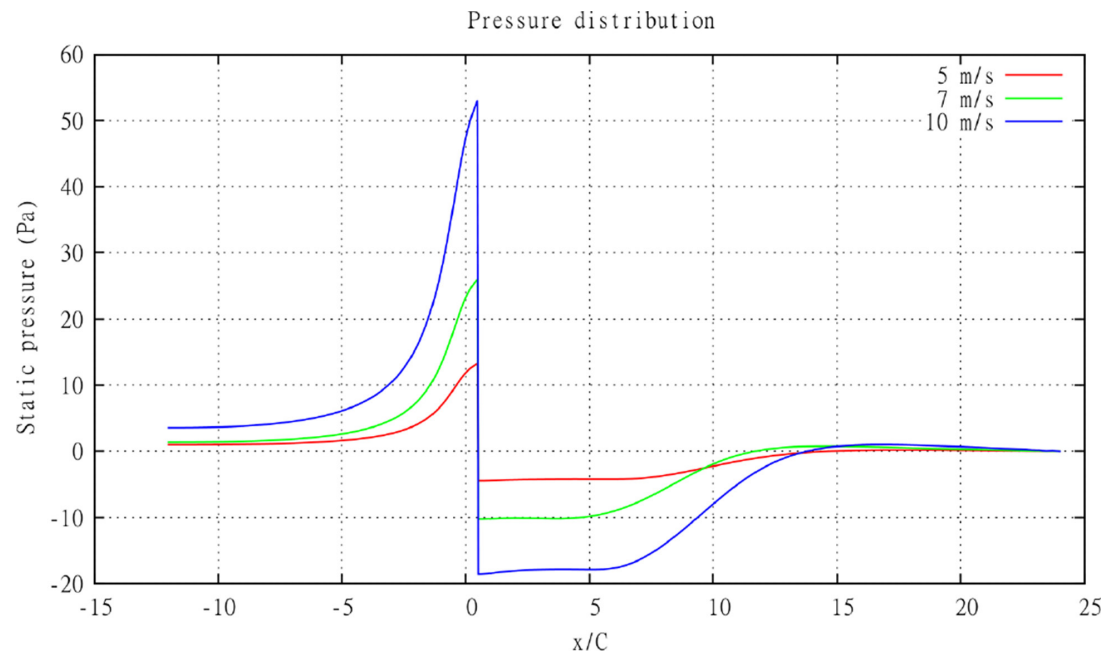

Figure 5: Centre-line pressure distribution for three different free stream velocities at different axial locations of the computational domain. 
downstream are not the same. Even if the boundary conditions at the inlet were set such that the static pressure is equal to zero, a slightly higher pressure is then computed since it is required to drive the flow past the high resistance disc. Tests showed that extending the computational domain (along both $\mathrm{x}$ and $\mathrm{y}$ directions) with the same DAWT geometry, the pressure gradient reduced but was still present.

The flow field around the diffuser with AD is presented in Figure 6 using contour of the stream-wise velocity, under free stream condition $U_{\infty}=10 \mathrm{~ms}^{-1}$. The figure is color-coded with $\mathrm{x}$-component velocity. In the figure the flow goes from left to right. The AD induces a strong resistance to the flow, which can be clearly recognized by the light blue contour upstream of the $\mathrm{AD}$. As expected, the flow just downstream of the $\mathrm{AD}$, from $\mathrm{x} / \mathrm{C}=0.5$, shows a further reduction and the velocity magnitude approaches zero indicated by the dark blue contour.

Figure 7 compares the computed axial and radial velocity profiles with experimental measurements at different $x$ and $y$ locations. The left-hand side of the Figure 7 shows the velocity distribution along the diffuser center-line for different $U_{\infty}$. As clearly visible, the computed stream-wise velocity component nicely follows the measurements showing good agreement in presence of the velocity jump across the AD. The velocity jump is to be expected because of the high $C_{T}$. The largest differences between experiments and computations are present downstream of the $\mathrm{AD}$ starting from $\mathrm{x} / \mathrm{C}=0.5$ to 1 . These differences are caused by the inaccuracy in the pitot tube measurements mainly due its size and locations of both the static and dynamic probes. However, the agreement is considered to be overall acceptable. The righthand side of Figure 7 projects a comparison between experiments and computations of velocity profiles along the AD radial direction, which is extracted just upstream as indicated in Figure. 6. As before a good agreement is reached.

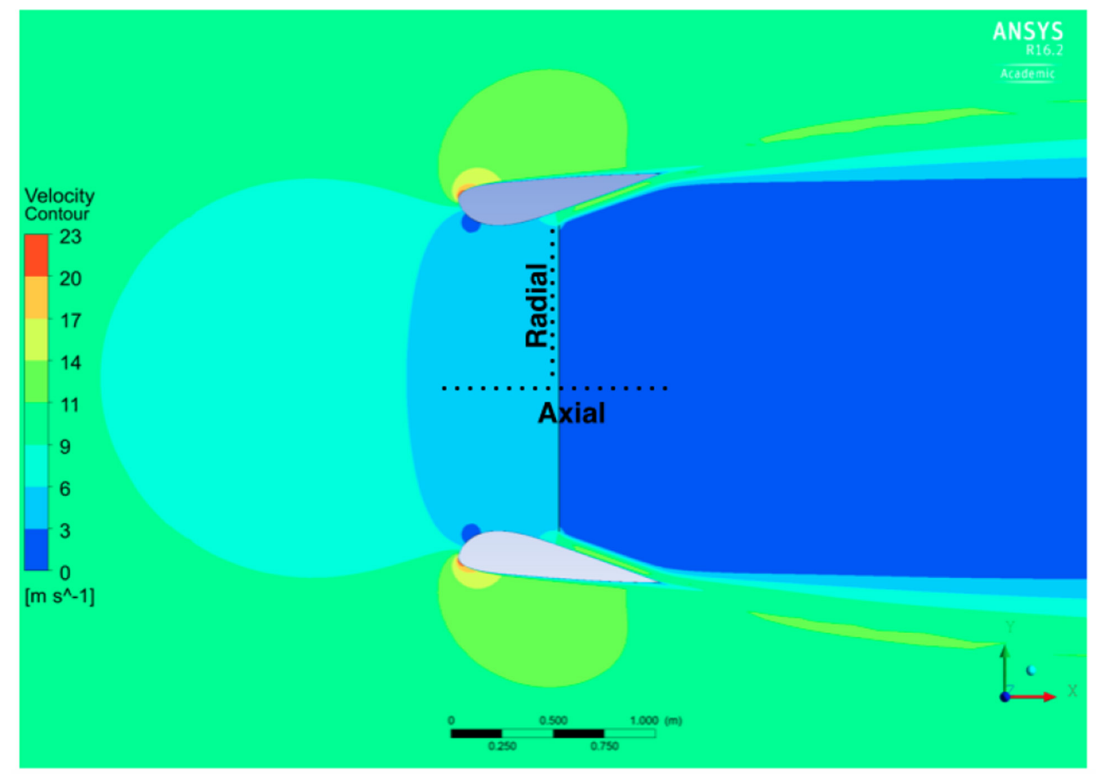

Figure 6: Stream-wise velocity contour plot around a DAWT using the AD force method, with an inlet speed of $10 \mathrm{~ms}^{-1}$. The dotted lines denote the position of axial and radial pitot-static tube measurements used for validation. 

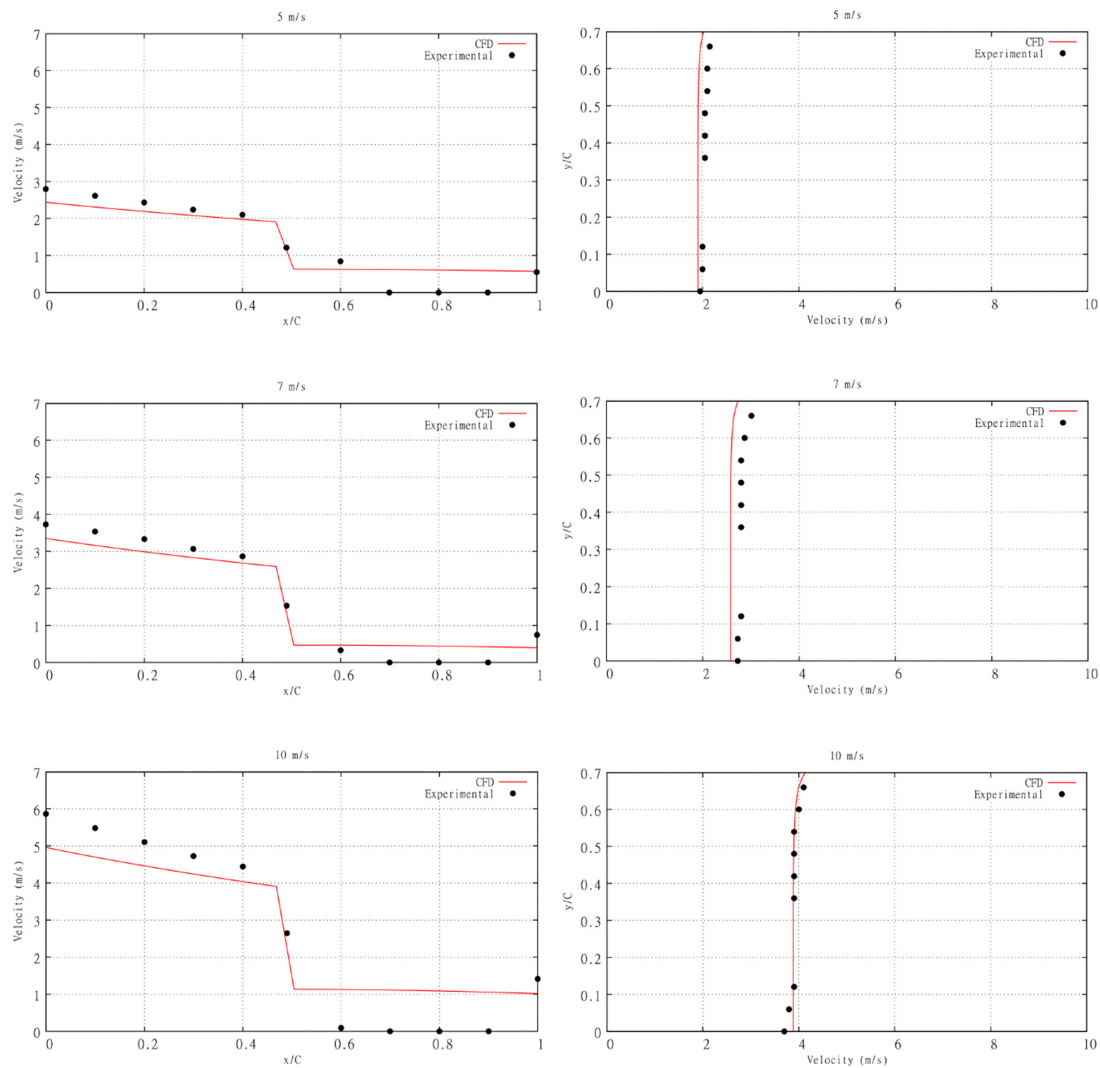

Figure 7: Comparison between CFD and experimental results with free stream velocity equal to $5,7 \& 10 \mathrm{~ms}^{-1}$. (left) Velocity distribution in the axial direction; (right) velocity distribution in the radial direction.

\section{CONCLUSION}

A computational approach to study of a diffuser augmented wind turbine has been presented. In this study, the AD inside the diffuser is modeled using discrete body forces. This approach of treating the body forces as pressure jump reduces significantly the number of cells needed to model the pressure jump. In the present research context, this can potentially lead to further study in DAWT modeling, and therefore, opening the possibility to carry performance analysis of the DAWT system. The results from the simulation show a good agreement with the experimental data while exhibiting some limitations, for example, the zero velocity in axial direction just behind the AD location. The need for more refined experimental data is clear with advanced measurement techniques like the particle image velocimetry (PIV), and this will form part of our future work.

\section{ACKNOWLEDGMENTS}

The work is sponsored by STW organization, grant number- 12728. Authors would like to acknowledge Juan Tang for providing the experimental data. 


\section{REFERENCES}

[1] Council, G.W.E., Wind is a global power source. Global Trend-GWEC, 2014.

[2] Foreman, K.M. \& Gilbert, B.L., Diffuser for Augmenting a Wind Turbine, US Patent 4,482,290, 1984.

[3] Ohya, Y., Karasudani, T., Sakurai, A., Abe, K.i. \& Inoue, M., Development of a shrouded wind turbine with a flanged diffuser. Journal of Wind Engineering and Industrial Aerodynamics, 96(5), pp. 524-539, 2008.

http://dx.doi.org/10.1016/j.jweia.2008.01.006

[4] Igra, O., Research and development for shrouded wind turbines. Energy Conversion and Management, 21(1), pp. 13-48, 1981.

http://dx.doi.org/10.1016/0196-8904(81)90005-4

[5] Van Bussel, G.J., The science of making more torque from wind: diffuser experiments and theory revisited. Journal of Physics: Conference Series, IOP Publishing, 75, p. 012010, 2007.

[6] Belloni, C., Hydrodynamics of Ducted and Open-Centre Tidal Turbines, Ph.D. thesis, University of Oxford, 2013.

[7] Thorpe, T., The advantages of ducted over unducted turbines. 6th European Wave and Tidal Energy Conference, Glasgow, UK, 2005.

[8] Phillips, D.G. An Investigation on Diffuser Augmented Wind Turbine Design, Ph.D. thesis, ResearchSpace@ Auckland, 2003.

[9] Gaden, D.L. \& Bibeau, E.L., A numerical investigation into the effect of diffusers on the performance of hydro kinetic turbines using a validated momentum source turbine model. Renewable Energy, 35(6), pp. 1152-1158, 2010.

http://dx.doi.org/10.1016/j.renene.2009.11.023

[10] National Aerospace Center, N.L., Design of small diffuser augmented wind turbine. Technical report, NLR Technical Paper, 2009.

[11] Mikkelsen, R., Actuator Disc Methods Applied to Wind Turbines, Ph.D. thesis, Technical University of Denmark, 2003.

[12] Crasto, G., Gravdahl, A., Castellani, F. \& Piccioni, E., Wake modeling with the actuator disc concept. Energy Procedía, 24, pp. 385-392, 2012.

http://dx.doi.org/10.1016/j.egypro.2012.06.122

[13] Harrison, M., Batten, W., Myers, L. \& Bahaj, A., Comparison between cfd simulations and experiments for predicting the far wake of horizontal axis tidal turbines. Renewable Power Generation, IET, 4(6), pp. 613-627, 2010. http://dx.doi.org/10.1049/iet-rpg.2009.0193

[14] Rethore, P.E.M. \& Sorensen, N.N., Actuator disc model using a modified rhie-chow/ simple pressure correction algorithm. comparison with analytical solutions. 2008 European Wind Energy Conference and Exhibition, 2008.

[15] Lignarolo, L., Ragni, D., Krishnaswami, C., Chen, Q., Ferreira, C.S. \& Van Bussel, G., Experimental analysis of the wake of a horizontal-axis wind-turbine model. Renewable Energy, 70, pp. 31-46, 2014.

http://dx.doi.org/10.1016/j.renene.2014.01.020

[16] Van Bussel, G.J., An assessment of the performance of diffuser augmented wind turbines (dawts). Proceedings of the 3rd ASME/JSME Joint Fluids Engineering Conference, San Francisco, California, 1999. 
[17] Laan, M.P., Sorensen, N.N., Rethore, P.E., Mann, J., Kelly, M.C. \& Troldborg, N., The $\mathrm{k}$-e-fp model applied to double wind turbine wakes using different actuator disk force methods. Wind Energy, 18(12), pp. 2223-2240, 2015. http://dx.doi.org/10.1002/we.1816

[18] Sanderse, B., Pijl, V.D.S. \& Koren, B., Review of computational fluid dynamics for wind turbine wake aerodynamics. Wind Energy, 14(7), pp. 799-819, 2011. http://dx.doi.org/10.1002/we.458

[19] Mercker, E. \& Wiedemann, J., On the correction of interference effects in open jet wind tunnels. Technical report, SAE Technical Paper, 1996. 